\title{
Cellular Tumor Suppression
}

National Cancer Institute

\section{Source}

National Cancer Institute. Cellular Tumor Suppression. NCI Thesaurus. Code C19099.

Cellular Tumor Suppression is thought to involve elimination of cells that have undergone malignant transformation by patrolling cytotoxic T cells, NK cells, and macrophages due to recognition of altered cell surface antigens on the cancer cells. 